\title{
On Laplacian spectrum of unitary Cayley graphs
}

\author{
S. PIRZADA \\ Department of Mathematics, University of \\ Kashmir, Srinagar, India \\ email: pirzadasd@kashmiruniversity.ac.in
}

\author{
Z. BARATI \\ Department of Mathematics, Kosar \\ University of Bojnord, Bojnord, Iran \\ email: za.barati87@gmail.com
}

\author{
M. AFKHAMI \\ Department of Mathematics, University \\ of Neyshabur, Neyshabur, Iran \\ email: mojgan.afkhami@yahoo.com
}

\begin{abstract}
Let $R$ be a commutative ring with unity $1 \neq 0$ and let $R^{\times}$ be the set of all unit elements of R. The unitary Cayley graph of $R$, denoted by $G_{R}=$ Cay $\left(R, R^{\times}\right)$, is a simple graph whose vertex set is $R$ and there is an edge between two distinct vertices $x$ and $y$ of $R$ if and only if $x-y \in R^{\times}$. In this paper, we determine the Laplacian and signless Laplacian eigenvalues for the unitary Cayley graph of a commutative ring. Also, we compute the Laplacian and signless Laplacian energy of the graph $G_{R}$ and its line graph.
\end{abstract}

\section{Introduction}

We consider finite commutative rings $R$ with unit element $1 \neq 0$. Let $R^{\times}$be the set of all unit elements of $R$. We know that an Artinian ring $R$ can be written as $R \cong R_{1} \times \cdots \times R_{t}$, where $R_{i}$ is a finite local ring with maximal ideal $\mathfrak{M}_{i}$, for all $1 \leqslant i \leqslant t$. This decomposition is unique up to permutation of factors. We

Computing Classification System 1998: G.2.2

Mathematics Subject Classification 2010: 05C22, 05C50, 05C76

Key words and phrases: unitary Cayley graph, Laplacian spectrum, signless Laplacian spectrum, Laplacian energy 
denote the (finite) residue field $\frac{R_{i}}{\mathfrak{M}_{i}}$ by $K_{i}$ and $f_{i}=\left|K_{i}\right|=\frac{\left|R_{i}\right|}{\left|\mathfrak{M}_{i}\right|}$. Also, assume that $f_{1} \leqslant f_{2} \leqslant \cdots \leqslant f_{t}$.

A simple graph $G$ consists of a vertex set $V(G)=\left\{v_{1}, v_{2}, \ldots, v_{n}\right\}$ and the edge set $\mathrm{E}(\mathrm{G})=\left\{\boldsymbol{e}_{1}, e_{2}, \ldots, e_{\mathrm{m}}\right\}$. We call $|\mathrm{V}(\mathrm{G})=\mathrm{n}|$ and $|\mathrm{E}(\mathrm{G})|=\mathrm{m}$, respectively, as the order and the size of the graph $G$. The complement of $G$, denoted by $\bar{G}$, is the graph whose vertex set is same as that of $G$ and two vertices are adjacent in $\bar{G}$ if and only if they are not adjacent in $G$. A complete graph on $n$ vertices is denoted by $K_{n}$. A graph $G$ is multipartite if its vertex set can be partitioned into non-empty subsets, called partite sets, such that no two vertices in the same part are adjacent. A multipartite graph is complete if every vertex of a partite set is adjacent to each vertex of the other partite sets. A complete multipartite graph with $k$ parts is denoted by $K_{n_{1}, n_{2}, \ldots, n_{k}}$ where $n_{i}$ is the number of vertices in the $i$-th part of the graph.

The join of two graphs $G_{1}$ and $G_{2}$, denoted by $G_{1} \vee G_{2}$, is the graph with vertex set $V\left(G_{1}\right) \cup V\left(G_{2}\right)$ and edge set $E\left(G_{1}\right) \cup E\left(G_{2}\right) \cup\left\{x y ; x \in V\left(G_{1}\right), y \in\right.$ $\left.V\left(G_{2}\right)\right\}$. The direct product of $G_{1}$ and $G_{2}$, denoted by $G_{1} \otimes G_{2}$, is the graph with vertex set $V\left(G_{1}\right) \times V\left(G_{2}\right)$ in which $\left(u_{1}, v_{1}\right)$ and $\left(u_{2}, v_{2}\right)$ are adjacent if $u_{1}$ and $u_{2}$ are adjacent in $G_{1}$ and $v_{1}$ and $v_{2}$ are adjacent in $G_{2}$. For other undefined notations and terminology from graph theory and spectral graph theory, the readers are referred to $[6,18]$.

The unitary Cayley graph of $R$, denoted by $G_{R}=\operatorname{Cay}\left(R, R^{\times}\right)$, is a (simple) graph whose vertex set is $R$ and two distinct vertices $x$ and $y$ of $R$ are adjacent if and only if $x-y \in R^{\times}$. Some recent results on unitary Cayley graphs can be seen in [16]. If $G=\mathbb{Z}_{n}$ is the finite cyclic group of order $n$ and the set $S$ consists of two elements, the standard generator of $G$ and its inverse, then the Cayley graph is the cycle $C_{n}$. More generally, the Cayley graphs of finite cyclic groups are exactly the circulant graphs. Some examples of unitary Cayley graphs are given in Figure 1.
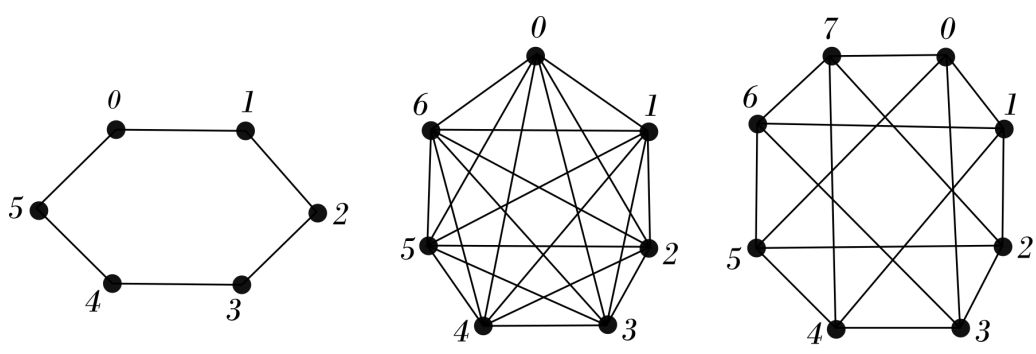

Figure 1: The unitary Cayley graphs for $\mathbb{Z}_{6}, \mathbb{Z}_{7}, \mathbb{Z}_{8}$ 
The following proposition is a basic consequence of the definition and it was illustrated in [1].

Proposition 1 Let $\mathrm{R}$ be a commutative ring.

(a) Then $\mathrm{G}_{\mathrm{R}}$ is a $\left|\mathrm{R}^{\times}\right|$-regular graph.

(b) If $\mathrm{R}$ is a local ring with maximal ideal $\mathfrak{M}$, then $\mathrm{G}_{\mathrm{R}}$ is a complete multipartite graph whose partite sets are the cosets of $\mathfrak{M}$ in $\mathrm{R}$. In particular, $\mathrm{G}_{\mathrm{R}}$ is a complete graph if and only if $\mathrm{R}$ is a field.

(c) If $\mathrm{R}$ is an Artinian ring and $\mathrm{R} \cong \mathrm{R}_{1} \times \ldots \times \mathrm{R}_{\mathrm{t}}$ as a product of local rings, then $\mathrm{G}_{\mathrm{R}} \cong \otimes_{i=1}^{\mathrm{t}} \mathrm{G}_{\mathrm{R}_{\mathrm{i}}}$. Hence, $\mathrm{G}_{\mathrm{R}}$ is a direct product of complete multipartite graphs.

The adjacency matrix $A$ of a graph $G$ is a $(0,1)$-square matrix of order $n$ whose $(i, j)$-entry is equal to 1 , if $v_{i}$ is adjacent to $v_{j}$ and equal to 0 , otherwise. The eigenvalues of $A$ are the eigenvalues of the graph $G$. The set of all eigenvalues of $\mathrm{G}$ is called the spectrum of $\mathrm{G}$. If $\lambda_{1} \geq \cdots \geq \lambda_{k}$ are the eigenvalues of $\mathrm{G}$ with multiplicities $r_{1}, \ldots, r_{k}$, respectively, the spectrum of $G$ is denoted by $\operatorname{Spec}(G)=\left(\begin{array}{ccc}\lambda_{1} & \ldots & \lambda_{k} \\ r_{1} & \ldots & r_{k}\end{array}\right)$. The energy of a graph was introduced by Gutman [13] and is defined as the sum of the absolute values of all the eigenvalues of a graph $\mathrm{G}$ and it is denoted by $\mathrm{E}(\mathrm{G})$.

Kiani et al. [15] obtained the following result about the eigenvalues of the unitary Cayley graph. Also, they computed the energy of the unitary Cayley graph of a finite commutative ring $R$.

Theorem 2 [15] Let $\mathrm{R}$ be a finite ring.

(a) If $\mathrm{R}$ is a finite local ring with the maximal ideal $\mathfrak{M}$ of size $\mathrm{m}$ and $\frac{|\mathrm{R}|}{\mathrm{m}}=\mathrm{f}$, then

$$
\operatorname{Spec}\left(\mathrm{G}_{\mathrm{R}}\right)=\left(\begin{array}{ccc}
\left|\mathrm{R}^{\times}\right| & 0 & -\mathrm{m} \\
1 & |\mathrm{R}|-\mathrm{f} & \mathrm{f}-1
\end{array}\right)
$$

In particular, if $\mathbb{F}_{\mathrm{q}}$ is the field with $\mathrm{q}$ elements, then

$$
\operatorname{Spec}\left(\mathrm{G}_{\mathbb{F}_{\mathrm{q}}}\right)=\left(\begin{array}{cc}
\mathrm{q}-1 & -1 \\
1 & \mathrm{q}-1
\end{array}\right)
$$


(b) Let $\mathrm{R}$ be a finite commutative ring, where $\mathrm{R} \cong \mathrm{R}_{1} \times \mathrm{R}_{2} \times \ldots \times \mathrm{R}_{\mathrm{t}}$ and $\mathrm{R}_{\mathrm{i}}$ is a local ring with maximal ideal $\mathfrak{M}_{i}$ of size $\mathrm{m}_{\mathrm{i}}$ for all $1 \leqslant i \leqslant \mathrm{t}$. Then the eigenvalues of $\mathrm{G}_{\mathrm{R}}$ are:

$(b-1)(-1)^{|C|} \frac{\left|R^{\times}\right|}{\prod_{j \in C}\left|R_{j}^{\times}\right| / m_{j}}$ with multiplicity $\prod_{j \in C}\left|R_{j}^{\times}\right| / m_{j}$ for all subsets $\mathrm{C}$ of the set $\{1,2, \ldots, \mathrm{t}\}$.

(b-2) 0 with multiplicity $|\mathrm{R}|-\prod_{i=1}^{\mathrm{t}}\left(1+\left|\mathrm{R}_{i}^{\times}\right| / \mathrm{m}_{\mathrm{i}}\right)$

Theorem 3 [15] Let $\mathrm{R} \cong \mathrm{R}_{1} \times \mathrm{R}_{2} \times \ldots \times \mathrm{R}_{\mathrm{t}}$ be a finite commutative ring where $\mathrm{R}_{\mathrm{i}}$ is a local ring for all $1 \leqslant \mathrm{i} \leqslant \mathrm{t}$. Then $\mathrm{E}\left(\mathrm{G}_{\mathrm{R}}\right)=2^{\mathrm{t}}\left|\mathrm{R}^{\times}\right|$.

Let $D(G)=\operatorname{diag}\left(d_{1}, d_{2}, \ldots, d_{n}\right)$ be the diagonal matrix associated to the graph $G$, where $d_{i}=\operatorname{deg}\left(v_{i}\right)$ is the degree of the vertex $v_{i}$, for all $1 \leqslant i \leqslant n$. The matrices $\mathrm{L}(\mathrm{G})=\mathrm{D}(\mathrm{G})-\mathrm{A}(\mathrm{G})$ and $|\mathrm{L}|(\mathrm{G})=\mathrm{D}(\mathrm{G})+\mathrm{A}(\mathrm{G})$ are respectively, called the Laplacian and the signless Laplacian matrices of $G$. Their spectrum are respectively, the Laplacian spectrum and the signless Laplacian spectrum of the graph $G$. We denote the Laplacian spectrum and the signless Laplacian spectrum of the graph $G$ by $\operatorname{Spec}_{L}(G)$ and $\operatorname{Spec}_{|\mathrm{L}|}(\mathrm{G})$, respectively. Both the matrices $\mathrm{L}(\mathrm{G})$ and $|\mathrm{L}|(\mathrm{G})$ are real symmetric, positive semi-definite and therefore their eigenvalues are non-negative real numbers. Let $0=\mu_{1} \leqslant \mu_{2} \leqslant \cdots \leqslant \mu_{n}$ and $\mu_{n}^{+} \leqslant \mu_{n-1}^{+} \leqslant \cdots \leqslant \mu_{1}^{+}$be respectively, the Laplacian spectrum and the signless Laplacian spectrum of G. It is known that the smallest eigenvalue of $L(G)$ is 0 with multiplicity equal to the number of connected components of G. So, $\mu_{2}>0$ if and only if $\mathrm{G}$ is connected. Also, the least eigenvalue of the signless Laplacian matrix of a connected graph is 0 if and only if the graph is bipartite. In this case, 0 is a simple eigenvalue. Furthermore, it is easy to see that $\operatorname{tr}(\mathrm{L}(\mathrm{G}))=\sum_{i=1}^{n} \mu_{i}=2 \mathrm{~m}$ and $\operatorname{tr}(-\mathrm{L}-(\mathrm{G}))=\sum_{i=1}^{n} \mu_{i}^{+}=2 \mathrm{~m}$. Recent work on Laplacian eigenvalues can be seen in $[2,5,9,10,11,12]$. The Laplacian energy of a graph $G$ defined by Gutman and Zhou [14] is $\operatorname{LE}(G)=\sum_{i=1}^{n}\left|\mu_{i}-\frac{2 m}{n}\right|$. The Laplacian energy, which is an extension of graph energy concept, has found remarkable chemical applications (see [24]). For recent development on $\operatorname{LE}(\mathrm{G})$ see $[7,8]$ and the references therein. The signless Laplacian energy $|L| E(G)$ of $G$, in analogy to $\operatorname{LE}(G)$, is defined as $|\mathrm{L}| \mathrm{E}(\mathrm{G})=\sum_{i=1}^{n}\left|\mu_{i}^{+}-\frac{2 m}{n}\right|$. Recent work on Laplacian eigenvalues can be seen in [19].

The rest of the paper is organized as follows. In Section 2, we determine the Laplacian spectrum and the Laplacian energy of the unitary Cayley graph $G_{R}$. Also, we completely obtain the signless Laplacian spectrum of the graph $G_{R}$ 
and compute the signless Laplacian energy of $G_{R}$. Further, we compute the Laplacian and signless Laplacian energy of the line graph of $G_{R}$.

\section{Laplacian spectrum of unitary Cayley graphs}

We begin with the following theorem, which gives the Laplacian spectrum of the join of two graphs $G_{1}$ and $G_{2}$.

Theorem 4 [17] Let $\mathrm{G}_{1}$ and $\mathrm{G}_{2}$ be two graphs with $\mathrm{n}_{1}$ and $\mathrm{n}_{2}$ vertices, respectively. Suppose that $0=\lambda_{1} \leqslant \lambda_{2} \leqslant \cdots \leqslant \lambda_{n_{1}}$ and $0=\mu_{1} \leqslant \mu_{2} \leqslant \cdots \leqslant \mu_{n_{2}}$ are the Laplacian eigenvalues of $\mathrm{G}_{1}$ and $\mathrm{G}_{2}$, respectively. Then the Laplacian eigenvalues of the graph $\mathrm{G}_{1} \vee \mathrm{G}_{2}$ are

(i) 0 with multiplicity 1 ,

(ii) $\lambda_{i}+n_{2}$ with multiplicity 1 for all $2 \leqslant i \leqslant n_{1}$,

(iii) $\mu_{j}+n_{1}$ with multiplicity 1 for all $2 \leqslant j \leqslant n_{2}$,

(iv) $\mathrm{n}_{1}+\mathrm{n}_{2}$ with multiplicity 1 .

Now, we have the following observation.

Lemma 5 If $\mathrm{G}=\mathrm{K}_{\mathrm{n}_{1}, \mathrm{n}_{2}, \ldots, \mathrm{n}_{\mathrm{k}}}$, where $\mathrm{n}_{\mathrm{i}} \in \mathbb{N}$ for all $1 \leqslant \mathrm{i} \leqslant \mathrm{k}$, then the Laplacian eigenvalues of $\mathrm{G}$ are

(i) 0 with multiplicity 1 ,

(ii) $\alpha_{i}=\sum_{\substack{j=1 \\ j \neq i}}^{k} n_{j}$ with multiplicity $n_{i}-1$ for all $1 \leqslant i \leqslant k$,

(iii) $\mathrm{n}_{1}+\mathrm{n}_{2}+\cdots+\mathrm{n}_{\mathrm{k}}$ with multiplicity $\mathrm{k}-1$.

Proof. We induct on $k$. For $k=2$, we have $G=\bar{K}_{n_{1}} \vee \bar{K}_{n_{2}}$. So, by Theorem 4 , we have that

$$
\operatorname{Spec}_{L}\left(K_{n_{1}, n_{2}}\right)=\left(\begin{array}{cccc}
0 & n_{1} & n_{2} & n_{1}+n_{2} \\
1 & n_{2}-1 & n_{1}-1 & 1
\end{array}\right) .
$$

Assume that the hypothesis is true for $K_{n_{1}, n_{2}, \ldots, n_{k}}$.

We prove it for the graph $K_{n_{1}, n_{2}, \ldots, n_{k}, n_{k+1}}$.

Clearly, $K_{n_{1}, n_{2}, \ldots, n_{k}, n_{k+1}} \cong K_{n_{1}, n_{2}, \ldots, n_{k}} \vee \bar{K}_{n_{k}}$.

Now, by Theorem 4 , it is easy to see that the Laplacian eigenvalues of $K_{n_{1}, n_{2}, \ldots, n_{k}, n_{k+1}}$ are 
(i) 0 with multiplicity 1 ,

(ii) $\sum_{\substack{j=1 \\ j \neq i}}^{k+1} n_{j}$ with multiplicity $n_{i}-1$ for all $1 \leqslant i \leqslant k+1$,

(iii) $n_{1}+n_{2}+\cdots+n_{k}+n_{k+1}$ with multiplicity $k$.

At first, we assume that $R$ is a local ring.

Proposition 6 Let $(\mathrm{R}, \mathfrak{M})$ be a local ring with $|\mathfrak{M}|=\mathrm{m}$ and $\left|\frac{\mathrm{R}}{\mathfrak{M}}\right|=\mathrm{f}$. Then

$$
\operatorname{Spec}_{\mathrm{L}}\left(\mathrm{G}_{\mathrm{R}}\right)=\left(\begin{array}{ccc}
0 & \left|\mathrm{R}^{\times}\right| & |\mathrm{R}| \\
1 & |\mathrm{R}|-\mathrm{f} & \mathrm{f}-1
\end{array}\right) \text {. }
$$

In particular, if $\mathrm{R}=\mathbb{F}_{\mathrm{q}}$ is the field with $\mathrm{q}$ elements, then

$$
\operatorname{Spec}_{\mathrm{L}}\left(\mathrm{G}_{\mathbb{F}_{\mathrm{q}}}\right)=\left(\begin{array}{cc}
0 & \mathrm{q} \\
1 & \mathrm{q}-1
\end{array}\right) \text {. }
$$

Proof. It is easy to see that $G_{R}$ is a complete multipartite graph in which every partite set is a coset of $\mathfrak{M}$. So, $G_{R}$ is the join of $f$ copies of the empty graph $\overline{\mathrm{K}}_{\mathrm{m}}$. Now, by Lemma 5, we have

$$
\operatorname{Spec}_{L}\left(G_{R}\right)=\left(\begin{array}{ccc}
0 & |R|-m & |R| \\
1 & |R|-f & f-1
\end{array}\right) .
$$

Since $|R|-m=\left|R^{\times}\right|$, therefore

$$
\operatorname{Spec}_{L}\left(G_{R}\right)=\left(\begin{array}{ccc}
0 & \left|R^{\times}\right| & |R| \\
1 & |R|-f & f-1
\end{array}\right)
$$

The Laplacian spectrum of the direct product of graphs has been described completely only when the factor graphs are regular. The Laplacian eigenvalues of the direct product of two regular graphs are listed in the following theorem.

Theorem 7 [3] Let $\mathrm{G}_{1}$ be an $\mathrm{r}_{1}$-regular graph with $\mathrm{n}_{1}$ vertices and $\mathrm{G}_{2}$ be an $\mathrm{r}_{2}$-regular graph with $\mathrm{n}_{2}$ vertices. Let $\operatorname{Spec}_{\mathrm{L}}\left(\mathrm{G}_{1}\right)=\left(\lambda_{1}, \lambda_{2}, \ldots, \lambda_{\mathrm{n}_{1}}\right)$ and $\operatorname{Spec}_{\mathrm{L}}\left(\mathrm{G}_{2}\right)=\left(\mu_{1}, \mu_{2}, \ldots, \mu_{n_{2}}\right)$. Then the eigenvalues of the graph $\mathrm{G}_{1} \otimes \mathrm{G}_{2}$ are $r_{1} \mu_{j}+r_{2} \lambda_{i}-\mu_{j} \lambda_{i}$ for all $1 \leqslant i \leqslant n_{1}$ and $1 \leqslant j \leqslant n_{2}$. 
In the following theorem, we obtain the Laplacian eigenvalues of $G_{R}$ with their multiplicities. Here, $\left|R_{S}^{\times}\right|$stands for $\left|R_{s_{1}}^{\times} \times R_{s_{2}}^{\times} \ldots \times R_{s_{k}}^{\times}\right|$, where $S=$ $\left\{s_{1}, \ldots, s_{k}\right\} \subseteq\{1,2, \ldots, t\}$ (if $S=\emptyset$, then we define $\left|R_{S}^{\times}\right|=1$ ).

Theorem 8 Let $\mathrm{R}$ be a finite commutative ring such that $\mathrm{R} \cong \mathrm{R}_{1} \times \mathrm{R}_{2} \times \cdots \times \mathrm{R}_{\mathrm{t}}$, where $\left(\mathrm{R}_{i}, \mathfrak{M}_{i}\right)$ is a local ring with $\left|\mathfrak{M}_{i}\right|=\mathrm{m}_{i}$ and $\left|\frac{R_{i}}{\mathfrak{M}_{i}}\right|=f_{i}$. Then the Laplacian eigenvalues of $\mathrm{G}_{\mathrm{R}}$ are

(i) 0 with multiplicity 1 ,

(ii) $\left|\mathrm{R}^{\times}\right|$with multiplicity $|\mathrm{R}|-\prod_{i=1}^{\mathrm{t}} \mathrm{f}_{i}$,

(iii) $\lambda_{\mathrm{A}}$ with multiplicity $\prod_{i \in \mathrm{A}^{\prime}}\left(\mathrm{f}_{\mathrm{i}}-1\right)$ for all $\mathrm{A} \subsetneq\{1,2, \ldots, \mathrm{t}\}$, where

$$
\lambda_{A}=\left|R_{A}^{\times}\right| \sum_{\substack{C=\left\{i_{1}, i_{2}, \ldots, i_{k}\right\} \subseteq A^{\prime} \\ k=1}}^{\left|A^{\prime}\right|}(-1)^{|C|-1}\left|R_{i_{1}}\right|\left|R_{i_{2}}\right| \ldots\left|R_{i_{k}}\right| \frac{\left|R_{A^{\prime}}^{\times}\right|}{\left|R_{C}^{\times}\right|}
$$

and $\mathrm{A}^{\prime}$ is the complement of $\mathrm{A}$.

Proof. We use induction on $t$. For $t=1$ and the local $\operatorname{ring} R \cong R_{1}$, by Proposition 6, we have

$$
\operatorname{Spec}_{L}\left(G_{R}\right)=\left(\begin{array}{ccc}
0 & \left|R^{\times}\right| & |R| \\
1 & |R|-f & f-1
\end{array}\right)
$$

Note that $\emptyset$ is the only proper subset of $\{1\}$ and $\lambda_{\emptyset}=|R|$. So, we are done in this case. Now, assume that the Laplacian eigenvalues of $R_{1} \times R_{2} \times \cdots \times R_{t-1}$ are

(i) 0 with multiplicity 1 ,

(ii) $\left|R_{1}^{\times} \times R_{2}^{\times} \times \cdots \times R_{t-1}^{\times}\right|$with multiplicity $\left|R_{1} \times R_{2} \times \cdots \times R_{t-1}\right|-\prod_{i=1}^{t-1} f_{i}$,

(iii) $\lambda_{A}$ with multiplicity $\prod_{i \in A^{\prime}}\left(f_{i}-1\right)$ for all $A \subsetneq\{1,2, \ldots, t-1\}$, where

$$
\lambda_{A}=\left|R_{A}^{\times}\right| \sum_{\substack{C=\left\{i_{1}, i_{2}, \ldots, i_{k}\right\} \subseteq A^{\prime} \\ k=1}}^{\left|A^{\prime}\right|}(-1)^{|C|-1}\left|R_{i_{1}}\right|\left|R_{i_{2}}\right| \ldots\left|R_{i_{k}}\right| \frac{\left|R_{A^{\prime}}^{\times}\right|}{\left|R_{C}^{\times}\right|} .
$$


Now, we determine the Laplacian eigenvalues of $G_{R}$ when $R \cong R_{1} \times R_{2} \times \cdots \times$ $R_{t-1} \times R_{t}$. We know that $G_{R} \cong G_{R_{1} \times R_{2} \times \cdots \times R_{t-1}} \otimes G_{R_{t}}$. Note that the graphs $G_{R_{1} \times R_{2} \times \cdots \times R_{t-1}}$ and $G_{R_{t}}$ are regular, so we can use Theorem 7 . Since

$$
\operatorname{Spec}_{L}\left(G_{R_{t}}\right)=\left(\begin{array}{ccc}
\mu_{1}=0 & \mu_{2}=\left|R_{t}^{\times}\right| & \mu_{3}=\left|R_{t}\right| \\
1 & \left|R_{t}\right|-f_{t} & f_{t}-1
\end{array}\right)
$$

we have the following cases to consider.

Case 1. For $\mu_{1}=0$, we have the following eigenvalues.

1.1. 0 with multiplicity 1 ,

1.2. $\left|R_{1}^{\times} \times R_{2}^{\times} \times \cdots \times R_{t-1}^{\times}\right| \times\left|R_{t}^{\times}\right|$with multiplicity $\left|R_{1} \times R_{2} \times \cdots \times R_{t-1}\right|-$ $\prod_{i=1}^{t-1} f_{i}$

1.3. $\lambda_{A} \times\left|R_{t}^{\times}\right|$with multiplicity $\prod_{i \in A^{\prime}}\left(f_{i}-1\right)$ for all $A \subsetneq\{1,2, \ldots, t-1\}$.

Case 2. For $\mu_{2}=\left|R_{t}^{\times}\right|$, we obtain the following eigenvalues.

2.1. $\left|R_{1}^{\times} \times R_{2}^{\times} \times \cdots \times R_{t-1}^{\times}\right|\left|R_{t}^{\times}\right|$with multiplicity $\left|R_{t}\right|-f_{t}$,

2.2. $\left|R_{1}^{\times} \times R_{2}^{\times} \times \cdots \times R_{t-1}^{\times}\right|\left|R_{t}^{\times}\right|$with multiplicity $\left(\left|R_{1} \times R_{2} \times \cdots \times R_{t-1}\right|-\right.$ $\left.\prod_{i=1}^{t-1} f_{i}\right)\left(\left|R_{t}\right|-f_{t}\right)$,

2.3. $\left|R_{1}^{\times} \times R_{2}^{\times} \times \cdots \times R_{t-1}^{\times} \| R_{t}^{\times}\right|$with multiplicity $\sum_{A \subsetneq\{1,2, \ldots, t-1\}} \prod_{i \in A^{\prime}}\left(f_{i}-\right.$ 1) $\left(\left|R_{t}\right|-f_{t}\right)$.

Therefore, in this case, we see that the eigenvalue is equal to $\mid R_{1}^{\times} \times R_{2}^{\times} \times$ $\cdots \times R_{t-1}^{\times}|| R_{t}^{\times} \mid$and this implies that $\left|R_{1}^{\times} \times R_{2}^{\times} \times \cdots \times R_{t-1}^{\times} \| R_{t}^{\times}\right|$is an eigenvalue with multiplicity $\left|R_{1} \times R_{2} \times \cdots \times R_{t-1}\right|\left(\left|R_{t}\right|-f_{t}\right)$.

Case 3. For $\mu_{3}=\left|R_{t}\right|$, the following eigenvalues can be obtained.

3.1. $\left|R_{1}^{\times} \times R_{2}^{\times} \times \cdots \times R_{t-1}^{\times}\right|\left|R_{t}\right|$ with multiplicity $f_{t}-1$,

3.2. $\left|R_{1}^{\times} \times R_{2}^{\times} \times \cdots \times R_{t-1}^{\times}\right|\left|R_{t}^{\times}\right|$with multiplicity $\left(\left|R_{1} \times R_{2} \times \cdots \times R_{t-1}\right|-\right.$ $\left.\prod_{i=1}^{t-1} f_{i}\right)\left(f_{t}-1\right)$

3.3. $\left|R_{1}^{\times} \times R_{2}^{\times} \times \cdots \times R_{t-1}^{\times}\right|\left|R_{t}\right|+\lambda_{A}\left|R_{t}^{\times}\right|-\lambda_{A}\left|R_{t}\right|$ with multiplicity $\left(\prod_{i \in A^{\prime}}\left(f_{i}-\right.\right.$ 1)) $\left(f_{t}-1\right)$ for all $A \subsetneq\{1,2, \ldots, t-1\}$.

Thus, we conclude the following.

(i) By case (1.1), 0 with multiplicity 1 is a Laplacian eigenvalue of $G_{R}$. 
(ii) By cases (1.2),(2.1), (2.2), (2.3) and (3.2), $\left|R_{1}^{\times} \times R_{2}^{\times} \times \cdots \times R_{t-1}^{\times} \| R_{t}^{\times}\right|$is a Laplacian eigenvalue of $G_{R}$. Its multiplicity is equal to

$$
\begin{aligned}
& \left(\left|R_{1} \times R_{2} \times \cdots \times R_{t-1}\right|-\prod_{i=1}^{t-1} f_{i}\right)+\left(\left|R_{1} \times R_{2} \times \cdots \times R_{t-1}\right|\left(\left|R_{t}\right|-f_{t}\right)\right)+ \\
& \left(\left|R_{1} \times R_{2} \times \cdots \times R_{t-1}\right|-\prod_{i=1}^{t-1} f_{i}\right)\left(f_{t}-1\right)=\left|R_{1} \times R_{2} \times \cdots \times R_{t}\right|-\prod_{i=1}^{t} f_{i}
\end{aligned}
$$

(iii) For $\mathfrak{A} \subsetneq\{1,2, \ldots, t\}$, three cases (1.3), (3.1) and (3.3) cover all eigenvalues with the type $\lambda_{\mathfrak{A}}$.

(a) From case (1.3), $\lambda_{A} \times\left|R_{t}^{\times}\right|$with multiplicity $\prod_{i \in A^{\prime}}\left(f_{i}-1\right)$ is a Laplacian eigenvalue of $G_{R}$ for all $A \subsetneq\{1,2, \ldots, t-1\}$. Note that if we set $\mathfrak{A}=A \cup\{\mathrm{t}\}$, then $\lambda_{\mathfrak{A}}=\lambda_{\mathrm{A}} \times\left|\mathrm{R}_{\mathrm{t}}^{\times}\right|$.

(b) From case (3.1), $\left|R_{1}^{\times} \times R_{2}^{\times} \times \cdots \times R_{t-1}^{\times}\right|\left|R_{t}\right|$ with multiplicity $f_{t}-1$ is a Laplacian eigenvalue of $G_{R}$. By setting $\mathfrak{A}=\{1,2, \ldots, t-1\}$, we have $\lambda_{\mathfrak{A}}=\left|R_{1}^{\times} \times R_{2}^{\times} \times \cdots \times R_{t-1}^{\times} \| R_{t}\right|$.

(c) From case (3.3), $\left|R_{1}^{\times} \times R_{2}^{\times} \times \cdots \times R_{t-1}^{\times}\right|\left|R_{t}\right|+\lambda_{A}\left|R_{t}^{\times}\right|-\lambda_{A}\left|R_{t}\right|$ with multiplicity $\left(\prod_{i \in A^{\prime}}\left(f_{i}-1\right)\right)\left(f_{t}-1\right)$ is a Laplacian eigenvalue of $G_{R}$, for all $A \subsetneq\{1,2, \ldots, t-1\}$. This case covers all eigenvalues like $\lambda_{\mathfrak{A}}$, when $\mathfrak{A}$ is a proper subset of the set $\{1,2, \ldots, t\}$ and $t \notin \mathfrak{A}$.

Now, we compute the Laplacian energy of the unitary Cayley graph, when $\mathrm{R}$ is a finite commutative ring. We start with the local case.

Lemma 9 Let $\mathrm{R}$ be a finite local commutative ring. Then $\operatorname{LE}\left(\mathrm{G}_{\mathrm{R}}\right)=2\left|\mathrm{R}^{\times}\right|$.

Proof. First, note that in the graph $G_{R}$, we have $\frac{2 m}{n}=\left|R^{\times}\right|$. Since the Laplacian spectrum of $G_{R}$ is

$$
\operatorname{Spec}_{L}\left(G_{R}\right)=\left(\begin{array}{ccc}
0 & \left|R^{\times}\right| & |R| \\
1 & |R|-f & f-1
\end{array}\right)
$$

we have $\operatorname{LE}\left(G_{R}\right)=2\left|R^{\times}\right|$.

Lemma 10 Let $\mathrm{R} \cong \mathrm{R}_{1} \times \mathrm{R}_{2}$, where $\left(\mathrm{R}_{1}, \mathfrak{M}_{1}\right)$ and $\left(\mathrm{R}_{2}, \mathfrak{M}_{2}\right)$ are local rings. Then

$$
\operatorname{LE}\left(G_{R}\right)=2^{2}\left|R^{\times}\right|
$$


Proof. We know that $G_{R} \cong G_{R_{1}} \otimes G_{R_{2}}$. Now, let $\operatorname{Spec}_{L}\left(G_{R_{1}}\right)=\left(\lambda_{1}, \lambda_{2}, \ldots, \lambda_{\left|R_{1}\right|}\right)$ and $\operatorname{Spec}_{\mathrm{L}}\left(\mathrm{G}_{\mathrm{R}_{2}}\right)=\left(\mu_{1}, \mu_{2}, \ldots, \mu_{\left|R_{2}\right|}\right)$. Then, by Theorem 7 , we have

$$
\begin{aligned}
\operatorname{LE}\left(G_{R}\right) & =\sum_{i=1}^{\left|R_{1}\right|} \sum_{j=1}^{\left|R_{2}\right|}|| R_{1}^{\times}\left|\mu_{j}+\right| R_{2}^{\times}\left|\lambda_{i}-\mu_{j} \lambda_{i}-\right| R_{1}^{\times}|| R_{2}^{\times}|| \\
& =\sum_{i=1}^{\left|R_{1}\right|} \sum_{j=1}^{\left|R_{2}\right|}\left|\left(\mu_{j}-\left|R_{2}^{\times}\right|\right)\right|\left|\left(\lambda_{i}-\left|R_{1}^{\times}\right|\right)\right| \\
& =\operatorname{LE}\left(G_{R_{1}}\right) \operatorname{LE}\left(G_{R_{2}}\right)=\left(2\left|R_{1}^{\times}\right|\right)\left(2\left|R_{2}^{\times}\right|\right)=2^{2}\left|R^{\times}\right| .
\end{aligned}
$$

Theorem 11 Let $\mathrm{R}$ be a finite commutative ring such that $\mathrm{R} \cong \mathrm{R}_{1} \times \mathrm{R}_{2} \times \cdots \times$ $\mathrm{R}_{\mathrm{t}}$, where $\mathrm{R}_{\mathrm{i}}$ is a local ring for all $1 \leqslant \mathrm{i} \leqslant \mathrm{t}$. Then $\mathrm{LE}\left(\mathrm{G}_{\mathrm{R}}\right)=2^{\mathrm{t}}\left|\mathrm{R}^{\times}\right|$.

Proof. This follows by using induction on $t$ and in view of Lemmas 9 and 10 .

The following results concern about the signless Laplacia spectrum of $G_{R}$. The proofs are omitted since they are similar to the proofs on the Laplacian spectrum.

Proposition 12 Let $(\mathrm{R}, \mathfrak{M})$ be a local ring with $|\mathfrak{M}|=\mathrm{m}$ and $\left|\frac{\mathrm{R}}{\mathfrak{M}}\right|=\mathrm{f}$. Then

$$
\operatorname{Spec}_{|\mathrm{L}|}\left(\mathrm{G}_{\mathrm{R}}\right)=\left(\begin{array}{ccc}
\left|\mathrm{R}^{\times}\right|-\mathrm{m} & \left|\mathrm{R}^{\times}\right| & 2\left|\mathrm{R}^{\times}\right| \\
\mathrm{f}-1 & |\mathrm{R}|-\mathrm{f} & 1
\end{array}\right) .
$$

In particular, if $\mathrm{R}=\mathbb{F}_{\mathrm{q}}$ is the field with $\mathrm{q}$ elements, then

$$
\operatorname{Spec}_{|\mathrm{L}|}\left(\mathrm{G}_{\mathbb{F}_{\mathrm{q}}}\right)=\left(\begin{array}{cc}
\mathrm{q}-2 & 2(\mathrm{q}-1) \\
\mathrm{q}-1 & 1
\end{array}\right) \text {. }
$$

Theorem 13 Let $\mathrm{R}$ be a finite commutative ring such that $\mathrm{R} \cong \mathrm{R}_{1} \times \mathrm{R}_{2} \times$ $\cdots \times R_{t}$, where $\left(R_{i}, \mathfrak{M}_{i}\right)$ is a local ring with $\left|\mathfrak{M}_{i}\right|=\mathrm{m}_{i}$ and $\left|\mathrm{R}_{i}\right|=\mathrm{f}_{i}$. Then the signless Laplacian eigenvalues of $\mathrm{G}_{\mathrm{R}}$ are

(i) $2\left|\mathrm{R}^{\times}\right|$with multiplicity 1 ,

(ii) $\left|\mathrm{R}^{\times}\right|$with multiplicity $|\mathrm{R}|-\prod_{i=1}^{\mathrm{t}} \mathrm{f}_{i}$,

(iii) $\lambda_{\mathrm{A}}$ with multiplicity $\prod_{i \in \mathrm{A}^{\prime}}\left(\mathrm{f}_{\mathrm{i}}-1\right)$ for all $\mathrm{A} \subsetneq\{1,2, \ldots, \mathrm{t}\}$ where

$$
\lambda_{A}=\left|R^{\times}\right|+(-1)^{\left|A^{\prime}\right|} \prod_{i \in A}\left|R_{i}^{\times}\right| \prod_{j \in A^{\prime}}\left|m_{j}\right| .
$$


If $R$ be a local finite commutative ring, it is easy to see that the signless Laplacian energy of $G_{R}$ is given by $|L| E\left(G_{R}\right)=2\left|R^{\times}\right|$. Further, if $R \cong R_{1} \times R_{2}$, where $R_{1}$ and $R_{2}$ are local rings, then $|L| E\left(G_{R}\right)=2^{2}\left|R^{\times}\right|$.

Thus, we have the following observation.

Theorem 14 Let $\mathrm{R}$ be a finite commutative ring such that $\mathrm{R} \cong \mathrm{R}_{1} \times \mathrm{R}_{2} \times \cdots \times$ $\mathrm{R}_{\mathrm{t}}$, $(\mathrm{t} \geqslant 2)$, where $\mathrm{R}_{\mathrm{i}}$ is a local ring for all $1 \leqslant \mathrm{i} \leqslant \mathrm{t}$. Then $|\mathrm{L}| \mathrm{E}\left(\mathrm{G}_{\mathrm{R}}\right)=2^{\mathrm{t}}\left|\mathrm{R}^{\times}\right|$.

Let $G$ be a graph with $n$ vertices and $m$ edges. The line graph $L(G)$ of $G$ is a simple graph whose vertex set is the set of edges of $G$ and two vertices of $\mathrm{L}(\mathrm{G})$ are adjacent if and only if the corresponding edges in $\mathrm{G}$ have a vertex in common. So, $n_{L(G)}$ (the number of vertices of $L(G)$ ) equals $m$. Also, it is easy to see that if $G$ is an $r$-regular graph, then $L(G)$ is a $(2 r-2)$-regular graph.

Theorem 15 [4] Let $\mathrm{G}$ be an $\mathrm{r}$-regular graph $(\mathrm{r} \geqslant 2)$ with $\mathrm{n}$ vertices and $\mathrm{m}$ edges. Then

(a) The Laplacian eigenvalues of the graph $\mathrm{L}(\mathrm{G})$ are

(i) $2-\lambda_{i}$, where $\lambda_{i}$ is a Laplacian eigenvalue of $\mathrm{G}$ for all $1 \leqslant i \leqslant n$,

(ii) $\mathrm{r}-2$ with multiplicity $\mathrm{m}-\mathrm{n}$.

(b) The signless Laplacian eigenvalues of the graph $\mathrm{L}(\mathrm{G})$ are

(i) $\lambda_{i}^{+}+2 r-4$, where $\lambda_{i}^{+}$is a signless Laplacian eigenvalue of $\mathrm{G}$ for all $1 \leqslant i \leqslant n$,

(ii) $2 \mathrm{r}-4$ with multiplicity $\mathrm{m}-\mathrm{n}$.

Now, we compute the Laplacian energy of the line graph of the unitary Cayley graphs. If $\left|R^{\times}\right|=1$, then $L\left(G_{R}\right)$ is an empty graph. So in this case, $\operatorname{LE}\left(L\left(G_{R}\right)\right)=0$. Thus, we suppose that $\left|R^{\times}\right| \geqslant 2$. Now, by Theorem 15 , the spectrum of $L(G)$ consists of the following eigenvalues.

(i) $2-\lambda_{i}$, where $\lambda_{i}$ is a Laplacian eigenvalue of $G_{R}$ for all $1 \leqslant i \leqslant|R|$,

(ii) $\left|\mathrm{R}^{\times}\right|-2$ with multiplicity $|\mathrm{R}|\left|\mathrm{R}^{\times}\right| / 2-|\mathrm{R}|$.

Proposition 16 Let $\mathrm{R}$ be a finite commutative ring with $\left|\mathrm{R}^{\times}\right| \geqslant 2$. Then

$$
\operatorname{LE}\left(\mathrm{L}\left(\mathrm{G}_{\mathrm{R}}\right)\right)=\frac{|\mathrm{R}|\left(\left|\mathrm{R}^{\times}\right|^{2}+4\left|\mathrm{R}^{\times}\right|-8\right)}{2} .
$$


Proof. Since $G_{R}$ is $\left|R^{\times}\right|$-reqular, $L\left(G_{R}\right)$ is a $\left(2\left|R^{\times}\right|-2\right)$-regular graph. So,

$$
2 \mathrm{~m}_{\mathrm{L}\left(\mathrm{G}_{\mathrm{R}}\right)} / \mathrm{n}_{\mathrm{L}\left(\mathrm{G}_{\mathrm{R}}\right)}=2\left|\mathrm{R}^{\times}\right|-2,
$$

where $n_{L\left(G_{R}\right)}$ and $m_{L\left(G_{R}\right)}$ are the number of vertices and edges of $L\left(G_{R}\right)$, respectively. We have

$$
\begin{aligned}
\operatorname{LE}\left(\mathrm{L}\left(\mathrm{G}_{\mathrm{R}}\right)\right) & \left.=\sum_{i=1}^{|\mathrm{R}|}\left|2-\lambda_{i}-\left(2\left|\mathrm{R}^{\times}\right|-2\right)\right|+\sum_{i=1}^{|\mathrm{R}|\left|\mathrm{R}^{\times}\right| / 2-|\mathrm{R}|}|| \mathrm{R}^{\times} \mid-2-\left(2\left|\mathrm{R}^{\times}\right|-2\right)\right) \mid \\
& =\sum_{i=1}^{|\mathrm{R}|}\left|-\lambda_{i}-2\right| \mathrm{R}^{\times}|+4|+\sum_{i=1}^{|\mathrm{R}|\left|\mathrm{R}^{\times}\right| / 2-|\mathrm{R}|}\left|\mathrm{R}^{\times}\right| \\
& \left.=\sum_{i=1}^{|\mathrm{R}|}\left(\lambda_{i}+2\left|\mathrm{R}^{\times}\right|-4\right)+\sum_{i=1}^{|\mathrm{R}|\left|\mathrm{R}^{\times}\right| / 2-|\mathrm{R}|}\left|\mathrm{R}^{\times}\right| \quad \quad \text { Since }\left|\mathrm{R}^{\times}\right| \geqslant 2\right) \\
& =\sum_{i=1}^{|\mathrm{R}|} \lambda_{i}+2|\mathrm{R}|\left|\mathrm{R}^{\times}\right|-4|\mathrm{R}|+\left(|\mathrm{R}|\left|\mathrm{R}^{\times}\right| / 2-|\mathrm{R}|\right)\left|\mathrm{R}^{\times}\right| \\
& =\left|\mathrm{R} \| \mathrm{R}^{\times}\right|+2|\mathrm{R}|\left|\mathrm{R}^{\times}\right|-4|\mathrm{R}|+\left(|\mathrm{R}|\left|\mathrm{R}^{\times}\right| / 2-|\mathrm{R}|\right)\left|\mathrm{R}^{\times}\right| \\
& \left(\operatorname{Since} \sum_{i=1}^{|\mathrm{R}|} \lambda_{i}=|\mathrm{R}|\left|\mathrm{R}^{\times}\right|\right) \\
& =\frac{|\mathrm{R}|\left(\left|\mathrm{R}^{\times}\right|^{2}+4\left|\mathrm{R}^{\times}\right|-8\right)}{2} .
\end{aligned}
$$

The following result gives the signless Laplacian energy of the line graph of unitary Cayley graphs. The proof is similar to the Laplacian case.

Proposition 17 Let $\mathrm{R}$ be a finite commutative ring with $\left|\mathrm{R}^{\times}\right| \geqslant 2$. Then

(i) If $\mathrm{f}_{1}=2$, then $|\mathrm{L}| \mathrm{E}\left(\mathrm{L}\left(\mathrm{G}_{\mathrm{R}}\right)\right)=2\left(|\mathrm{R}|\left(\left|\mathrm{R}^{\times}\right|-2\right)+1\right)$.

(ii) $|\mathrm{L}| \mathrm{E}\left(\mathrm{L}\left(\mathrm{G}_{\mathrm{R}}\right)\right)=2|\mathrm{R}|\left(\left|\mathrm{R}^{\times}\right|-2\right)$, otherwise. 


\section{References}

[1] R. Akhtar, M. Boggess, T. Jackson-Henderson, I. Jiménez, R. Karpman, A. Kinzel, D. Pritikin, On the unitary Cayley graph of a finite ring, Electron. J. Combin. 16 (2009) R117. $\Rightarrow 253$

[2] A. Alhevaz, M. Baghipur, Hilal A. Ganie, S. Pirzada, Brouwer type conjecture for the eigenvalues of distance signless Laplacian matrix of a graph, Linear Multilinear Algebra 69, 13 (2021) 2423-2440. $\Rightarrow 254$

[3] S. Barik, R. B. Bapat, S. Pati, On the Laplacian spectra of product graphs, Appl. Anal. Discrete Math. 9 (2015) 39-58. $\Rightarrow 256$

[4] S. Barik, D. Kalita, S. Pati, G. Sahoo, Spectra of graphs resulting from various graph operations and products: a survey, Spec. Matrices 6 (2018) 323-342. $\Rightarrow$ 261

[5] B. A. Rather, S. Pirzada, T. A. Naikoo, Y. Shang, On Laplacian eigenvalues of the zero-divisor graph associated to the ring of integers modulo n, Mathematics $\mathbf{9}, \mathbf{5}(2021) 482 . \Rightarrow 254$

[6] D. Cvetkovic, M. Doob, H. Sachs, Spectra of Graphs-Theory and Application, Academic Press, New York, 1980. $\Rightarrow 252$

[7] H. A. Ganie, S. Pirzada, A. Iványi, Energy, Laplacian energy of double graphs and new families of equienergetic graphs, Acta Univ. Sapientiae, Inform. 6 (2014) 89-117. $\Rightarrow 254$

[8] H. A. Ganie, S. Pirzada, E. T. Baskoro, On energy, Laplacian energy and p-fold graphs, Electron. J. Graph Theory Appl. 3 (2015) 94-107. $\Rightarrow 254$

[9] H. A. Ganie, S. Pirzada, R. Ul Shaban, X. Li, Upper bounds for the sum of Laplacian eigenvalues of a graph and Brouwer's conjecture, Discrete Math. Algorithms Appl. 11, 2 (2019) 195008 (15 pages). $\Rightarrow 254$

[10] H. A. Ganie, S. Pirzada, B. A. Rather, V. Trevisan, Further developments on Brouwer's conjecture for the sum of Laplacian eigenvalues of graphs, Linear Algebra Appl. $\mathbf{5 8 8}(2020)$ 1-18. $\Rightarrow 254$

[11] H. A. Ganie, S. Pirzada, V. Trevisan, On the sum of k largest Laplacian eigenvalues of a graph and clique number, Mediterranean J. Mathematics 18 (2021) Article No. 15 . $\Rightarrow 254$

[12] H. A. Ganie, S. Pirzada, B. A. Rather, R. Ul Shaban, On Laplacian eigenvalues of graphs and Brouwer's conjecture, J. Ramanujan Math. Soc. 36,1 (2021) 13-21. $\Rightarrow 254$

[13] I. Gutman, The Energy of a Graph: Old and New Results, Algebraic Combinatorics and Applications (Springer, Berlin), 2001. $\Rightarrow 253$

[14] I. Gutman, B. Zhou, Laplacian energy of a graph, Linear Algebra Appl. 414 (2006) 29-37. $\Rightarrow 254$

[15] D. Kiani, M. M. H. Aghaei, Y. Meemark, B. Suntornpoch, Energy of unitary Cayley graphs and gcd-graphs, Linear Algebra Appl. 435 (2011) 1336-1343. $\Rightarrow$ 253, 254

[16] W. Klotz, T. Sander, Some properties of unitary Cayley graphs, Electron. J. Combin. 14 (2007) Research Paper 45, 12 pages. $\Rightarrow 252$ 
[17] R. Merris, Laplacian graph eigenvectors, Linear Algebra Appl. 278 (1998) 221$236 . \Rightarrow 255$

[18] S. Pirzada, An Introduction to Graph Theory, Universities Press, Hyderabad, India, 2012. $\Rightarrow 252$

[19] S. Pirzada, H. A. Ganie, A. Alghamdi, On the sum of signless Laplacian spectra of graphs, Carpathian Math. Publications 11, 2 (2019) 407-417. $\Rightarrow 254$

Received: October 4, 2021 - Revised: October 11, 2021 